\title{
Molecular pathogenesis of primary central nervous system lymphoma
}

\author{
Cigall Kadoch, Patrick Treseler, M.D., Ph.D., and James L. Rubenstein, M.D., Ph.D. \\ Department of Molecular and Cell Biology, University of California, Berkeley; \\ Division of Hematology/Oncology and Department of Pathology, University of California, \\ San Francisco, California
}

\begin{abstract}
$\checkmark$ Primary central nervous system lymphoma (PCNSL) is an aggressive form of non-Hodgkin lymphoma (NHL) typically associated with a worse prognosis than other localized extranodal lymphomas with similar histological characteristics. The defining feature of PCNSL is its confinement to the central nervous system (CNS), with proclivity for growth within the leptomeningeal as well as intraocular compartments. Primary CNS lymphoma rarely disseminates outside the CNS and accounts for less than 5\% of all primary brain neoplasms. At least $95 \%$ of PCNSLs are of large B-cell histology, the most common subtype of NHL. Consistent with the trend seen in systemic NHLs, the incidence of PCNSL has markedly increased over the past three decades, both in immunocompromised and immunocompetent patients. Because PCNSL is relatively rare, the identification of molecular prognostic biomarkers and the definition of a standard therapeutic strategy have been challenging. The authors discuss the current knowledge of the molecular pathogenesis of CNS lymphomas and review the recent advances in gene expression profile analysis and identification of novel prognostic biomarkers.
\end{abstract}

\section{KEY WORDS • brain neoplasms • non-Hodgkin lymphoma $\quad$ molecular pathology $・$ gene expression • chemokine}

$\mathrm{P}$ RIMARY CNS lymphoma is a rare form of NHL that occurs within the craniospinal axis and accounts for less than $5 \%$ of all primary brain tumors. It is defined as lymphoma occurring in the brain, leptomeninges, spinal cord, or eyes without evidence of lymphoma outside the CNS. The majority of PCNSL tumors are highgrade B-cell lymphomas, despite the fact that B-cells are absent from normal brain. The tumors are most often diagnosed by stereotactic brain biopsy, which is often preceded by glucocorticoid therapy, administered to reverse neurological deficits. Because the majority of PCNSLs are sensitive to glucocorticoid agents and exhibit resolution and apoptosis in response to these medications, histopathological diagnosis and classification of PCNSL cases can be difficult. More than $90 \%$ of PCNSL tumors ultimately are diagnosed as high-grade, CD20-positive, diffuse large B-cell NHLs; the remaining $10 \%$ of cases include Burkitt, Burkitt-like, and lymphoblastic B-cell lymphomas as well as T-cell lymphomas. In up to $20 \%$ of cases, the diagnosis of PCNSL may be established by the combination of cytological findings of malignancy in the CSF and the exclusion of systemic lymphoma by bone marrow biopsy and axial imaging. However, cytological analysis of CSF and specimens from intravitreal compartments is associated with extremely poor sensitivity.

Abbreviations used in this paper: AIDS = acquired immunodeficiency syndrome; $\mathrm{CSF}=$ cerebrospinal fluid; $\mathrm{CNS}=$ central nervous system; $\mathrm{EBV}=$ Epstein-Barr virus; $\mathrm{NHL}=$ non-Hodgkin lymphoma; PCNSL = primary CNS lymphoma.
Because of the relative rarity of this type of brain tumor, as well as the difficulty of obtaining intracranial samples, there is a paucity of clinical material for molecular analysis of CNS lymphomas. Nevertheless, genetic studies are beginning to elucidate features of the molecular pathogenesis of these tumors. There is now evidence that CNS lymphomas exhibit a distinct gene expression profile compared with nodal lymphomas of the same histological type. In addition, emerging data indicate that CNS lymphomas are derived from a distinct cell of origin and exhibit a unique immunophenotype. Current efforts are focused on the identification of tumor biomarkers to facilitate noninvasive diagnosis as well as prognosis. The purpose of this review is to highlight key features and recent advances in our understanding of the molecular pathogenesis of PCNSL.

\section{Incidence}

During the past 30 years, the incidence of PCNSL has increased dramatically in all age groups. There were 2.5 cases per 10 million population in 1973 and 30 cases per 10 million population in $1992 ; ;^{10}$ in 1990 , PCNSL composed nearly $7 \%$ of all primary brain tumors. ${ }^{23} \mathrm{~A}$ primary factor responsible for the increase during this period has been the human immunodeficiency virus epidemic, which began in the 1980s. ${ }^{11}$ Patients with AIDS develop PCNSL at a rate 3600 -fold higher than the general population and have a lifetime risk of CNS lymphoma that approaches $20 \%$. It is important to note, however, that the incidence 
of PCNSL within immunocompetent populations has also increased rapidly during this interval, from 4 per 10 million in 1982 to 28 per 10 million in $1989 .{ }^{11}$ This increasing incidence of disease among immunocompetent individuals, which appears to be independent of overall trends in the incidence of brain tumors and NHL, has outpaced the milder increase in systemic lymphomas and remains essentially unexplained. . $^{8,12,30,32}$

\section{Gross Pathological Characteristics}

In the majority of cases, patients with PCNSL present with symptoms referable to an intracranial mass lesion. Often PCNSL lesions are periventricular, thus facilitating leptomeningeal seeding. Most patients present with a solitary lesion; multiple tumors are seen somewhat more often in AIDS-related cases. ${ }^{30}$ The lesions most commonly occur in the frontal and temporoparietal lobes, but may also be found in the basal ganglia; PCNSL lesions occur less frequently in the cerebellum, brainstem, and spinal cord. In approximately $20 \%$ of PCNSL cases, evidence of malignancy is found on CSF examination at the time of diagnosis, reflecting the propensity of these tumors for leptomeningeal dissemination. On gross macroscopic examination, PCNSLs range from well-circumscribed masses sometimes resembling metastases to ill-defined and highly infiltrative malignancies that are impossible to distinguish anatomically from adjacent normal brain tissue. The tumors often extend across the corpus callosum and involve both cerebral hemispheres. In at least $40 \%$ of cases, there is gross infiltration into the subarachnoid space. ${ }^{17,43}$

\section{Microscopic Pathological Characteristics}

Pathological assessment of tumors in PCNSL cases reveals a unique angiocentric pattern of tumor infiltration. Large B-cell lymphomas occurring outside the CNS do not exhibit particular growth around blood vessels. Histological staining techniques reveal a concentric network of reticulin fibers, which are particularly prominent in the perivascular lymphoid tissue. In some cases, lymphomas grow as diffuse sheets invading the surrounding brain parenchyma, meninges, or walls of ventricles. Typically, PCNSLs are highly infiltrative and usually extend significantly beyond the margins of the radiographically evident tumor. ${ }^{17}$

Routine immunophenotyping data from adequate pathology specimens demonstrate that the vast majority of PCNSLs $(>95 \%)$ are high-grade B-cell lymphomas in patients with AIDS as well as in immunocompetent patients. ${ }^{13,43}$ The majority of these tumors are diffuse large Bcell lymphomas, the most common form of NHL. Lowgrade primary CNS B-cell lymphomas are rare and are composed mainly of small lymphocytes. ${ }^{6}$ Although T-cell lymphomas have been reported as primary CNS tumors, they are infrequent in PCNSL, accounting for less than 5\% of all cases. ${ }^{15,26,31,38,40,44}$ Unlike B-cell lymphomas, most peripheral (nonlymphoblastic) T-cell lymphomas lack a distinctive morphological appearance, and consist of small, intermediate, or large cells, in variable combinations. Given this heterogeneous composition, which may suggest reactive T-cell proliferations, definitive diagnosis depends on clinical history, unequivocal T-cell phenotype and clear cytological demonstration of malignancy, overt infiltration with tissue destruction, or molecular evidence of a clonal T-cell population. In general, T-cell lymphomas have a poor prognosis, with an average 5-year survival rate of less than $30 \% .{ }^{38}$ Like B-cell CNS lymphomas, T-cell tumors also may exhibit a pattern of angiotropic growth around cerebral vessels. However, in contrast to B-cell PCNSLs, the majority of T-cell PCNSLs present in the posterior fossa. ${ }^{44}$

Approximately $20 \%$ of patients with PCNSL present with intraocular involvement at diagnosis; these tumors typically involve the vitreous, retina, and choroid. Patients who present with isolated intraocular lymphoma are at high risk for CNS dissemination; approximately $80 \%$ of these patients eventually will experience progression of CNS disease. It should be pointed out that lymphomatous involvement of the conjunctiva and orbit is not considered CNS disease; lymphomas in these sites exhibit a distinct pattern of growth and should be managed as extranodal manifestations of systemic lymphoma. ${ }^{38}$

Hodgkin lymphoma occurs only extremely rarely as a primary CNS lymphoma and usually is seen in the peripheral lymph nodes. ${ }^{29}$ Diagnosis of Hodgkin lymphoma is dependent on the identification of Reed-Sternberg cells or mononuclear variants demonstrating the characteristic CD30+ CD15+ immunophenotype, which can be seen against a background of benign-appearing inflammatory cells. ${ }^{20}$ Primary CNS Hodgkin disease may present as dural-based masses mimicking meningioma; these typically display the nodular sclerosis subtype of Hodgkin lymphoma. ${ }^{2,3,19}$ Prognosis of primary CNS Hodgkin disease is at present not well-defined, but appears to be associated with survival of between of 8 to 24 months following irradiation. ${ }^{2,3,19,29}$

\section{Tumor Pathogenesis}

In immunosuppressed individuals, such as patients with AIDS or patients treated with immunosuppressive agents in the setting of organ transplantation, PCNSL is usually linked to latent infection of the neoplastic B-cells by EBV. ${ }^{14}$ Infection of normal B-cells may result in their immortalization; however, proliferation of EBV-infected B-cells is usually suppressed by normal T-cell immunity. With diminished T-cell function, however, the EBV-infected clone may progress to malignant lymphoma. ${ }^{35,41}$ Moreover, EBV infection correlates with CNS tropism of lymphoma. The risk of CNS tumor dissemination in EBVpositive systemic AIDS-related NHL is approximately 10fold higher than the risk in EBV-negative cases. ${ }^{9}$ In addition, concordance of the presence of EBV DNA in the CSF as measured by polymerase chain reaction and suggestive abnormalities on neuroimaging may permit the presumptive diagnosis of CNS lymphoma in patients with AIDS. The biological basis for EBV-driven CNS tropism in lymphoma remains to be defined. ${ }^{1}$

A rat model of EBV-related PCNSL has been described based on stereotactic implantation of EBV-transformed human lymphoblastic B-cell lines into the caudate nucleus of the nude rat. This model has been used to explore the preclinical efficacy of antiviral therapy in the treatment of patients with simultaneous EBV infection and PCNSL. ${ }^{36}$ 
The CNS is an immunologically privileged site, normally devoid of B-cells, and the origin of the tumor cells in PCNSL has yet to be completely elucidated. One proposed mechanism postulates that a malignant clone of Bcells arising systemically might express specific adhesion molecule(s) that facilitate homing to the CNS, where the tumor cells proliferate and undergo secondary mutations in the absence of regulatory control by the immune system. To date, however, no differences in the expression of adhesion molecules have been established between PCNSL and systemic lymphomas. . $^{38,18,34}$

Chemokines represent an important class of molecules that regulate the trafficking of leukocytes as well as their proliferation and adhesion, contributing to the development of normal lymph node structure. Recently, a B-cellattracting chemokine, BCA-1 (CXCL-13) was shown to be expressed at significant levels in PCNSL tumors. A lymphoid chemokine, BCA-1 promotes B-cell homing to secondary lymphoid organs. It is expressed in Helicobacter pylori-induced mucosa-associated lymphoid tissue as well as in gastric lymphoma. ${ }^{22}$ Binding of BCA-1 to its receptor, CXCR5, also shown to be expressed by B-cells in PCNSL, may be involved in the extranodal localization of CNS lymphomas. Although BCA-1 has been demonstrated to be expressed by lymphoma cells, it does not appear to be produced by tumor endothelia in PCNSL and therefore may not contribute to the unique angiotropic pattern of growth in these tumors. ${ }^{39}$ Expression of the B-cell chemokines BCA-1 and SDF-1 by the retinal pigment epithelium has been demonstrated in primary intraocular lymphoma. Ectopic expression of these chemokines within the intraocular compartment may be significant in the attraction and homing of lymphoma to the retinal pigment epithelium from the choroidal circulation. . $^{738}$

A recent gene expression analysis using microarrays has defined for the first time molecular distinctions between PCNSL and nodal lymphomas of the large B-cell type. A variety of evidence has demonstrated that systemic large-cell lymphomas can be subclassified into three molecular types: germinal center, activated B-cell, and type 3, with the first subtype being associated with favorable prognosis. Primary CNS lymphomas have been shown to express features of each of these molecular subtypes; however, at least 100 genes were shown to be significantly differentially upregulated in cases of brain lymphoma. ${ }^{37}$ Among these genes were some associated with the unfolded protein response pathway, such as X-box binding protein 1 . The unfolded protein response pathway is a stress response pathway that may facilitate tumor adaptation in conditions of glucose deprivation. ${ }^{33}$ In addition, the protooncogenes $M Y C$ and PIMI also were shown to be expressed at high levels in these brain tumors. Evidence of somatic mutation of PIMI and $M Y C$ has also been found in PCNSL. ${ }^{25}$

In addition, ectopic expression of the B-cell growth factor interleukin-4 was also recently demonstrated in PCNSL. ${ }^{37}$ Interleukin-4 is not only a potential autocrine growth factor for lymphoma cells but may also function as a significant paracrine growth factor given its unique expression by tumor-associated endothelia in PCNSL. Interleukin-4 is not expressed in the vasculature of normal brain or the vasculature in malignant astrocytic neoplasms. These findings support the hypothesis that inteleukin-4 may be important in the pathogenesis and progression of PCNSL and may contribute to the angiotropic growth pattern of lymphoma cells within the CNS. In addition, the activated form of the transcription factor STAT6, a mediator of interleukin-4-dependent gene expression, was also shown to be expressed by tumor cells and tumor endothelia in PCNSL, providing an independent line of evidence for a significant functional role of interleukin-4 signaling in the tumorigenesis of this type of lymphoma. High-density tumors in which the activated form of STAT6 is found both in the lymphoma cells and the associated endothelia have been reported to be associated with early progression and short overall survival in patients treated with highdose methotrexate. The STAT6 expression pattern thus constitutes a novel biomarker for prognostic determination at the time of diagnosis of PCNSL.

\section{Molecular Pathological Findings and Immunophenotype}

Determination of the sequence of the immunoglobulin variable region genes in CNS lymphomas has revealed high frequencies of somatic mutations; these findings suggest that PCNSLs are derived from mature B-cells that have been exposed to antigen and have undergone T-celldependent affinity maturation in a germinal center microenvironment. ${ }^{24}$ In support of this conclusion is the fact that BCL-6, a germinal center marker, is expressed in the majority of PCNSLs that are not associated with AIDS. The expression of BCL-6 demonstrated by immunohistochemical analysis in CNS lymphomas has been shown to correlate retrospectively with favorable overall survival. ${ }^{4}$ Further characterization of the immunophenotype of PCNSLs has revealed that these tumors have overlapping features of germinal center and activated B-cell differentiation: immunohistochemical analyses have demonstrated that the majority of brain lymphomas express MUM-1, a marker of activated B-cells, and BCL-6, a marker of germinal centers. ${ }^{5,21}$ This conclusion was also suggested by results of microarray analysis of PCNSL gene expression. ${ }^{37}$ These results have significant implications in that they provide an explanation for how a subtype of large Bcell lymphoma that exhibits germinal center features and that commonly expresses BCL-6 is, in general, associated with an inferior outcome relative to systemic lymphomas of the same histological characteristics and stage.

There is significantly less information regarding the molecular pathogenesis of PCNSL in comparison with systemic lymphomas. One established molecular lesion in PCNSL that has been reproducibly identified is homozygous deletion or promoter hypermethylation of the CDKN2A gene, which produces P14ARF. Growth arrest induced by P14ARF is p53-dependent as p14ARF binds to MDM2, a mediator of p53 stabilization. Mutations of TP53 are rare in PCNSL, in contrast to systemic lymphomas in which inactivation of the TP53 gene may be detected in 20 to $40 \%$ of tumors. These findings suggest that early alterations of $C D K N 2 A$ in PCNSL facilitate tumor development and minimize the selection pressure for secondary mutations of TP53. ${ }^{28,38}$

Comparative genomic hybridization has also identified potentially significant genetic lesions in PCNSL. For example, gains have been detected on chromosome 12 
and the long arms of chromosome 1,18 , and 7 , while reduced DNA copy number was detected on chromosome 6 and on the short arms of chromosomes 17 and 18. Gain on chromosome 12 is the most frequent alteration identified by comparative genomic hybridization (in particular, gain in a region of $12 \mathrm{q}$, a locus known to contain the $M D M 2$, CDK4, and GLII). ${ }^{16,28,38}$

Allelic deletions of the long arm of chromosome 6 are frequent genomic aberrations in systemic lymphoma and acute lymphoblastic leukemias. The presence of $6 \mathrm{q}$ deletions is associated with adverse outcome in follicular lymphoma. ${ }^{42}$ Deletions of $6 \mathrm{q}$ in PCNSL occur in $66 \%$ of specimens. Reduced expression of PTPRK, a candidate tumor suppressor protein, was shown to correlate with 6q22-23 deletion, raising the possibility that this tyrosine phosphatase may modulate PCNSL progression. Loss of heterozygosity analysis also suggests that aberrations on $6 \mathrm{q}$ predict short survival in PCNSL. ${ }^{27}$ In addition, deletion or promoter methylation of $C D K N 2 A, R B 1$, and $C D K N 1 B$ have been identified in PCNSL, but these do not appear to correlate with survival. ${ }^{4,28,38}$

\section{References}

1. Antinori A, De Rossi G, Ammassari A, Cingolani A, Murri R, Di Giuda D, et al: Value of combined approach with thallium201 single-photon emission computed tomography and Epstein-Barr virus DNA polymerase chain reaction in CSF for the diagnosis of AIDS-related primary CNS lymphoma. J Clin Oncol 17:554-560, 1999

2. Ashby MA, Barber P, Holmes AE, Freer CE, Collins RD: Primary intracranial Hodgkin's disease. A case report and discussion. Am J Surg Pathol 12:294-249, 1988

3. Biagi J, MacKenzie R, Lim MS, Sapp M, Berinstein N: Primary Hodgkin's disease of the CNS in an immunocompetent patient: a case study and review of the literature. Neuro-oncol 2: 239-243, 2000

4. Braaten KM, Betensky RA, de Leval L, Okada Y, Hochberg FH, Louis DN, et al: BCL-6 expression predicts improved survival in patients with primary central nervous system lymphoma. Clin Cancer Res 9:1063-1069, 2003

5. Camilleri-Broet S, Criniere E, Broet P, Delwail V, Mokhtari K, Moreau A, et al: A uniform activated B-cell-like immunophenotype might explain the poor prognosis of primary central nervous system lymphomas: analysis of 83 cases. Blood 107: 190-196, 2006

6. Cantoni S, Oreste P, Nosari AM, Schiantarelli C, Caroli Costantini M, Landonio G, et al: Low-grade primary central nervous system lymphoma in HIV-positive patients: report of two cases. Eur J Haematol 59:59-60, 1997

7. Chan CC, Buggage RR, Nussenblatt RB: Intraocular lymphoma. Curr Opin Ophthalmol 13:411-418, 2002

8. Ciacci JD, Tellez C, VonRoenn J, Levy RM. Lymphoma of the central nervous system in AIDS. Semin Neurol 19:213-221, 1999

9. Cingolani A, Gastaldi R, Fassone L, Pierconti F, Giancola ML, Martini M, et al: Epstein-Barr virus infection is predictive of CNS involvement in systemic AIDS-related non-Hodgkin's lymphomas. J Clin Oncol 18:3325-3330, 2000

10. Corn BW, Marcus SM, Topham A, Hauck W, Curran WJ JR: Will primary central nervous system lymphoma be the most frequent brain tumor diagnosed in the year 2000? Cancer 79:2409-2413, 1997

11. Cote TR, Manns A, Hardy CR, Yellin FJ, Hartge P: Epidemiology of brain lymphoma among people with or without acquired immunodeficiency syndrome. AIDS/Cancer Study Group. J Natl Cancer Inst 88:675-679, 1996

12. Eby NL, Grufferman S, Flannelly CM, Schold SC Jr, Vogel FS,
Burger PC: Increasing incidence of primary brain lymphoma in the US. Cancer 62:2461-2465, 1988

13. Flinn IW, Ambinder R: AIDS primary central nervous system lymphoma. Curr Opin Oncol 8:373-376, 1996

14. Forsyth PA, DeAngelis LM: Biology and management of AIDS-associated primary CNS lymphomas. Hematol Oncol Clin North Am 10:1125-1134, 1996

15. Grove A, Vyberg M: Primary leptomeningeal T-cell lymphoma: a case and a review of primary T-cell lymphoma of the central nervous system. Clin Neuropathol 12:7-12, 1993

16. Harada K, Nishizaki T, Kubota H, Harada K, Suzuki M, Sasaki $\mathrm{K}$ : Distinct primary central nervous system lymphoma defined by comparative genomic hybridization and laser scanning cytometry. Cancer Genet Cytogenet 125:147-150, 2001

17. Jellinger KA, Paullus W: Primary central nervous system lymphomas-an update. J Cancer Res Clin Oncol 119:7-27, 1992

18. Jellinger KA, Paullus W: Primary central nervous system lymphomas-new pathological developments. J Neurooncol 24: 33-36, 1995

19. Johnson MD, Kinney MC, Scheithauer BW, Briley RJ, Hamilton K, McPherson WF, et al: Primary intracerebral Hodgkin's disease mimicking meningioma: case report. Neurosurgery 47:454-456, 2000

20. Klein R, Mullges W, Bendszus M, Woydt M, Kreipe H, Roggendorf W: Primary intracerebral Hodgkin's disease: report of a case with Epstein-Barr virus association and review of the literature. Am J Surg Pathol 23:477-481, 1999

21. Lin CH, Kuo KT, Chuang SS, Kuo SH, Chang JH, Chang KC, et al: Comparison of the expression and prognostic significance of differentiation markers between diffuse large B-cell lymphomas of central nervous system origin and peripheral nodal origin. Clin Cancer Res 12:1152-1156, 2006

22. Mazzucchelli L, Blaser A, Kappeler A, Scharli P, Laissue JA, Baggiolini M, et al: BCA-1 is highly expressed in Helicobacter pylori-induced mucosa-associated lymphoid tissue and gastric lymphoma. J Clin Invest 104:49-54, 1999

23. Miller DC, Hochberg FH, Harris NL, Gruber ML, Louis DN, Cohen H: Pathology with clinical correlations of primary central nervous system non-Hodgkin's lymphoma. The Massachusetts General Hospital experience 1958-1989. Cancer 74:1383-1397, 1994

24. Montesinos-Rongen M, Kuppers R, Schluter D, Spieker T, Van Roost D, Schaller C, et al: Primary central nervous system lymphomas are derived from germinal-center B cells and show a preferential usage of the V4-34 gene segment. Am J Pathol 155:2077-2086, 1999

25. Montesinos-Rongen M, Van Roost D, Schaller C, Wiestler OD, Deckert M: Primary diffuse large B-cell lymphomas of the central nervous system are targeted by aberrant somatic hypermutation. Blood 103:1869-1875, 2004

26. Morgello S, Maiese K, Petito CK: T-cell lymphoma in the CNS: clinical and pathologic features. Neurology 39:1190-1196, 1989

27. Nakamura M, Kishi M, Sakaki T, Hashimoto H, Nakase H, Shimada K, et al: Novel tumor suppressor loci on 6q22-23 in primary central nervous system lymphomas. Cancer Res 63:737-741, 2003

28. Nakamura M, Shimada K, Ishida E, Konishi N: Histopathology, pathogenesis and molecular genetics in primary central nervous system lymphomas. Histol Histopathol 19:211-219, 2004

29. Nakayama H, Tokuuye K, Kagami Y, Sumi M, Murayama S, Kawashima M, et al: Brain involvement in Hodgkin's disease: case reports and review of the literature. Radiat Med 18:205-208, 2000

30. Newell ME, Hoy J, Cooper SG, DeGraaff B, Grulich AE, Bryant $\mathrm{M}$, et al: Human immunodeficiency virus-related primary central nervous system lymphoma: factors influencing survival in 111 patients. Cancer 100:2627-2636, 2004 
31. Nitta T, Uda K, Ebato M, Ikezaki K, Fukui M, Sato K: Primary peripheral-postthymic T-cell lymphoma in the central nervous system: immunological and molecular approaches to diagnosis. J Neurosurg 82:77-82, 1995

32. Olson JE, Janney CA, Rao RD, Cerhan JR, Kurtin PJ, Schiff D, et al: The continuing increase in the incidence of primary central nervous system non-Hodgkin lymphoma: a surveillance, epidemiology, and end results analysis. Cancer 95:1504-1510, 2002

33. Park HR, Tomida A, Sato S, Tsukumo Y, Yun J, Yamori T, et al: Effect on tumor cells of blocking survival response to glucose deprivation. J Natl Cancer Inst 96:1300-1310, 2004

34. Paullus W, Jellinger K: Comparison of integrin adhesion molecules expressed by primary brain lymphomas and nodal lymphomas. Acta Neuropathol 86:360-364, 1993

35. Penn I: The role of immunosuppression in lymphoma formation. Springer Semin Immunopathol 20:343-355, 1998

36. Roychowdhury S, Peng R, Baiocchi RA, Bhatt D, Vourganti S, Grecula J, et al: Experimental treatment of Epstein-Barr virusassociated primary central nervous system lymphoma. Cancer Res 63:965-971, 2003

37. Rubenstein JL, Fridlyand J, Shen A, Aldape K, Ginzinger D, Batchelor T, et al: Gene Expression and Angiotropism in Primary CNS Lymphoma. Blood 107:3716-3723, 2006

38. Rubenstein JL, Treseler P, O'Brien JM: Pathology and genetics of primary central nervous system and intraocular lymphoma. Hematol Oncol Clin North 19:705-717, 2005

39. Smith JR, Braziel RM, Paoletti S, Lipp M, Uguccioni M, Rosenbaum J: Expression of B-cell-attracting chemokine 1 (CXCL13) by malignant lymphocytes and vascular endothelium in primary central nervous system lymphoma. Blood 101: 815-821, 2003

40. Stark AM, Tiemann M, Dorner L, Melnikowa E, Mehdorn HM,
Blomer U: Primary peripheral T-cell lymphoma of the central nervous system. Zentralbl Neurochir 65:191-194, 2004

41. Thomas JA, Crawford DH, Burke M: Clinicopathologic implications of Epstein-Barr virus related B cell lymphoma in immunocompromised patients. J Clin Pathol 48:287-290, 1995

42. Tilly H, Rossi A, Stamatoullas A, Lenormand B, Bigorgne C, Kunlin A, et al: Prognostic value of chromosomal abnormalities in follicular lymphoma. Blood 84:1043-1049, 1994

43. Tomlinson FH, Kurtin PJ, Suman VJ, Scheithauer BW, O'Fallon JR, Kelly PJ, et al: Primary intracerebral malignant lymphoma: a clinicopathological study of 89 patients. J Neurosurg 82:558-566, 1995

44. Villegas E, Villa S, Lopez-Guillermo A, Petit J, Ribalta T, Graus F: Primary central nervous system lymphoma of T-cell origin: description of two cases and review of the literature. J Neurooncol 34:157-161, 1997

This work was supported by a National Cancer Institute Research Career Award and a University of California, San Francisco, Brain Tumor SPORE (Specialized Programs of Research Excellence) Award (J.L.R.).

Manuscript received September 1, 2006.

Accepted in final form October 4, 2006.

Address reprint requests to: James L. Rubenstein, M.D, Ph.D., Assistant Professor of Medicine, Division of Hematology/Oncology, University of California, San Francisco, Room M1282 Box 1270, San Francisco, California 94143. email: jamesr@medicine. ucsf.edu. 